\title{
Mucinous cystadenocarcinoma of the breast, case report
}

\author{
Victor E Valdespino $^{1 *}$, Israel Lopez Matamoros ${ }^{1}$, Miguel Lopez Valle${ }^{1}$, Mara Mayorga Rangel ${ }^{1}$, Hilda Mendoza Ramon ${ }^{2}$ and Victor \\ Valdespino Gomez ${ }^{3}$ \\ ${ }^{1}$ Department of Gynecology and Oncology, Gynecological and Obstetrician Hospital 4, "Luis Castelazo Ayala” Social Security Mexican Institute, Mexico City, Mexico \\ ${ }^{2}$ Department of Pathology, Gynecological and Obstetrician Hospital 4, "Luis Castelazo Ayala" Social Security Mexican Institute, Mexico City, Mexico \\ ${ }^{3}$ Department of Surgical Oncology, Gynecological and Obstetrician Hospital 4, "Luis Castelazo Ayala” Social Security Mexican Institute, Mexico City, Mexico
}

\begin{abstract}
The WHO categorizes the breast tumors in 21 distinct histological types based on cell morphology and architecture. A widely accepted fact that the aggressive nature of the breast cancer can be determined by its histological type, grade, nodal status, and metastasis holds the ground even in the era of immunohistochemistry (IHC) and molecular.

An extremely rare variant of primary breast carcinoma belongs to the family of mucin-producing carcinoma sharing the same histology as the mucinous cystadenocarcinoma of the ovary and pancreas. Similar to our case, most of these tumors are reported in postmenopausal females aged between 47 and 96 years.

Due to the rarity of these tumors, there is an extreme paucity of literature on this topic. Most of the literature available is in the form of case reports. We show a 68 years old patient with a breast tumor, with a starting biopsy papillary proliferation disease, a tumorectomy reported phyllodes with mucinous cystadenocarcinoma with narrow border after that, the patient was programmed a radical modify mastectomy with nodal axillary dissection. She is alive without adjuvant treatment afterward for 21 months.
\end{abstract}

\section{Introduction}

The primary mucinous cystadenocarcinoma of the breast is a very infrequent tumor. The most common sites of mucinous cystadenocarcinoma include the ovary, pancreas, and appendix. Less than 25 cases of primary mucinous cystadenocarcinoma of the breast have been reported. The first report was made by Koenig published the first 4 cases in 1998 [1]. Mucinous cystadenocarcinoma in the breast is found in postmenopausal women, and this tumor present as a relatively large, semi tender or firm, cystic mass with a favorable prognosis and long survival [2].

Another diagnostic peril is misdiagnosing mucinous cystadenocarcinoma as primary invasive mucinous carcinoma of the breast, a far more common lesion than mucinous cystadenocarcinoma. Histologically, unlike the cells of invasive mucinous carcinoma, cells of mucinous cystadenocarcinoma possess abundant intracellular mucin and maintain this feature in the extravasated stromal mucin as well. Also, mucinous cystadenocarcinoma is essentially negative for estrogen receptor and progestogen receptor whereas, invasive mucinous carcinoma is fundamentally positive for estrogen receptor and progestogen receptor. In the breasts, mucin-producing tumors are classified as mucinous cystadenocarcinoma, signet ring cell carcinoma, or mucocele-like tumors, frequently is necessary to perform immunochemistry techniques to get a specific diagnosis [3].

Performing a correct diagnosis is difficult, immunohistochemistry, and precise diagnosis with hematoxylin and eosin die is required. Sometimes it is necessary to take whole surgical specimen for an accurate diagnosis; not only a biopsy.

\section{Case report}

Female patient, 68 years old, with diabetes with 25 years on treatment, arterial hypertension 30 years on medical treatment. First pregnant at 20 years old and for 1 year she gave breastfeeding, in total 8 deliveries. Six months previous to first medical evaluation, tumor was noticed in the right breast. In medical oncologic evaluation, we founded a neoplasm $4 \mathrm{~cm}$, near to areola, in 3 hours in the right breast. The tumor founded is irregular, very hard, and 1 nodal with metastasis suspect in the right armpit.

Mastography reported, a great irregular shaped calcification, BIRADS 4B. Core biopsy reported proliferative disease of breast with papillar frame.

After that, we proposed to perform an excisional tumorectomy as the pathological report of breast showed cystadenocarcinoma mucinous in $60 \%$ and phyllodes tumor, low grade in $40 \%$, the maximum extension was $5.5 \mathrm{~cm}$. Limits were free at $1 \mathrm{~mm}$.

With narrow tumor limit we suggest a radical modify mastectomy, and radical axillary dissection, this pathological report was 15 nodal free of disease, tumoral bed, and limits without tumor, areola without tumor, microcalcification in benign breast tissue.

The patient did not receive adjuvant treatment, she was only in observation with clinical evaluation every 4 months, until now she is free of relapse with a survival of 21 months.

${ }^{*}$ Correspondence to: Victor E Valdespino, Department of Gynecology and Oncology, Gynecological and Obstetrician Hospital 4, "Luis Castelazo Ayala" Social Security Mexican Institute, Mexico City, Mexico, E-mail: edvaldespinocg@ yahoo.com

Key words: cystadenocarcinoma, metastasis, progesterone, eostrogen

Received: November 15, 2019; Accepted: November 22, 2019; Published: November 25, 2019 
Cytokeratin 7 positive, cytokeratin 20 negative, estrogen receptor was negative, progestogen receptor-negative, her-2- neu negative (Figures 1-5).

\section{Discussion}

Comparison of mucinous-producing tumors in the breast are: Mucocele like a tumor, mucinous carcinoma, signet ring cell carcinoma, and mucinous cystadenocarcinoma of the breast, the diagnosis has to be made cautiously because this tumor has specific immunochemical characteristics and histopathological differences.

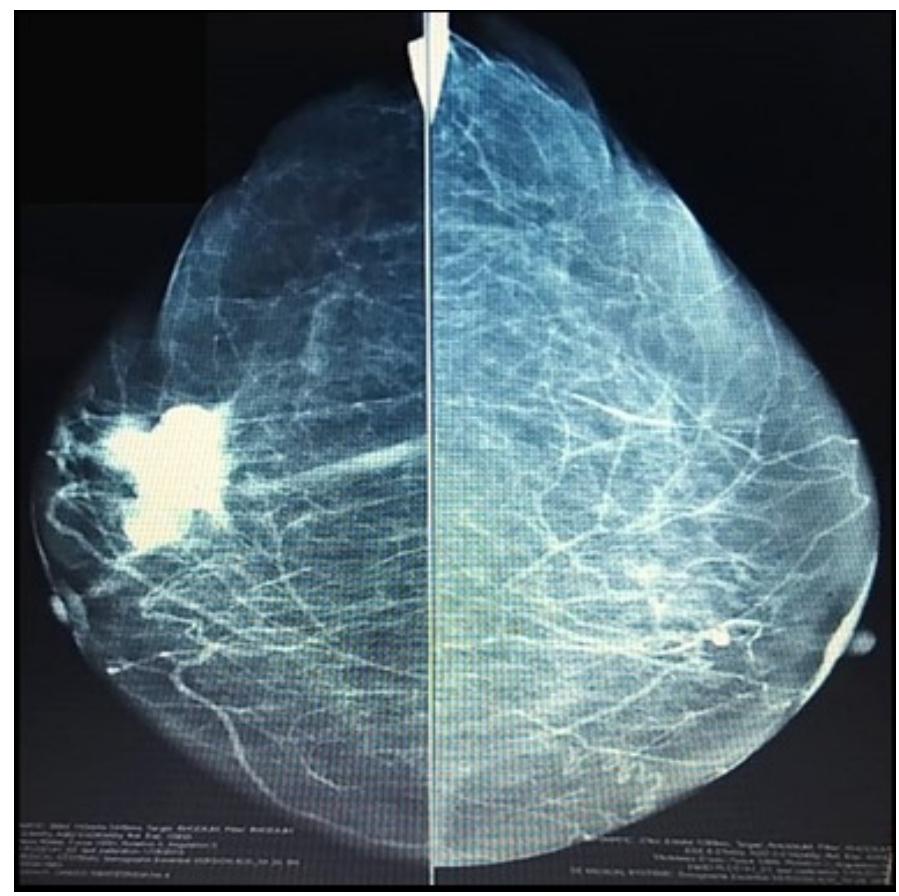

Figure 1. Mastography

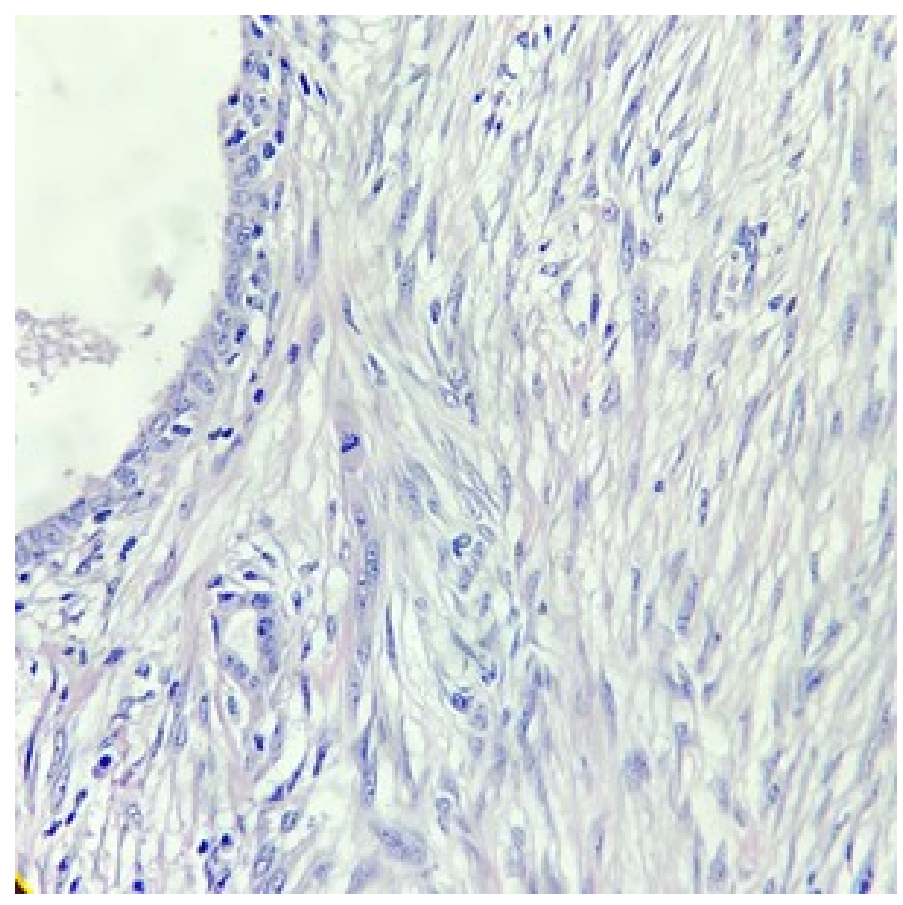

Figure 2. Phyllodes tumor

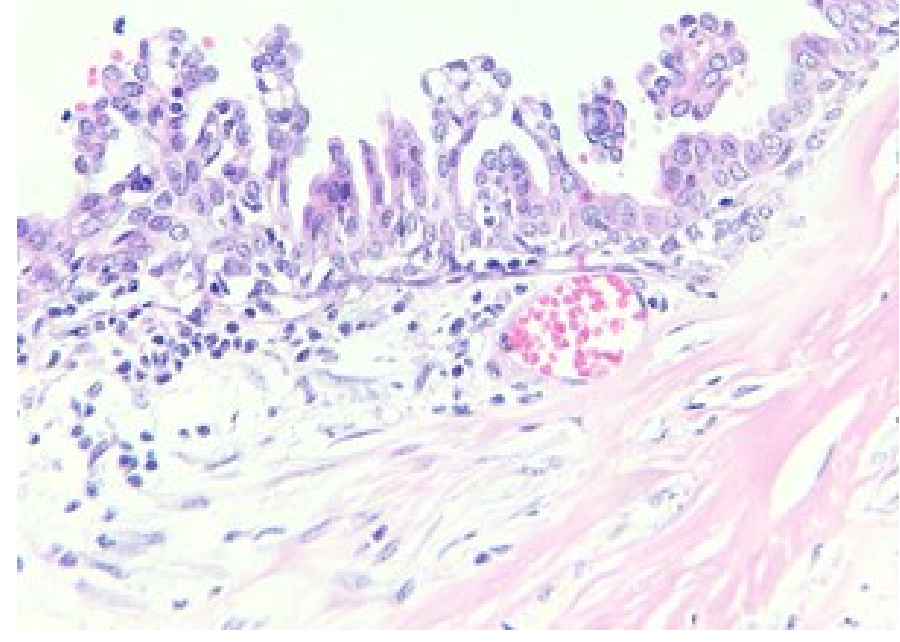

Figure 3. Mucinous cystadenocarcinoma of breast

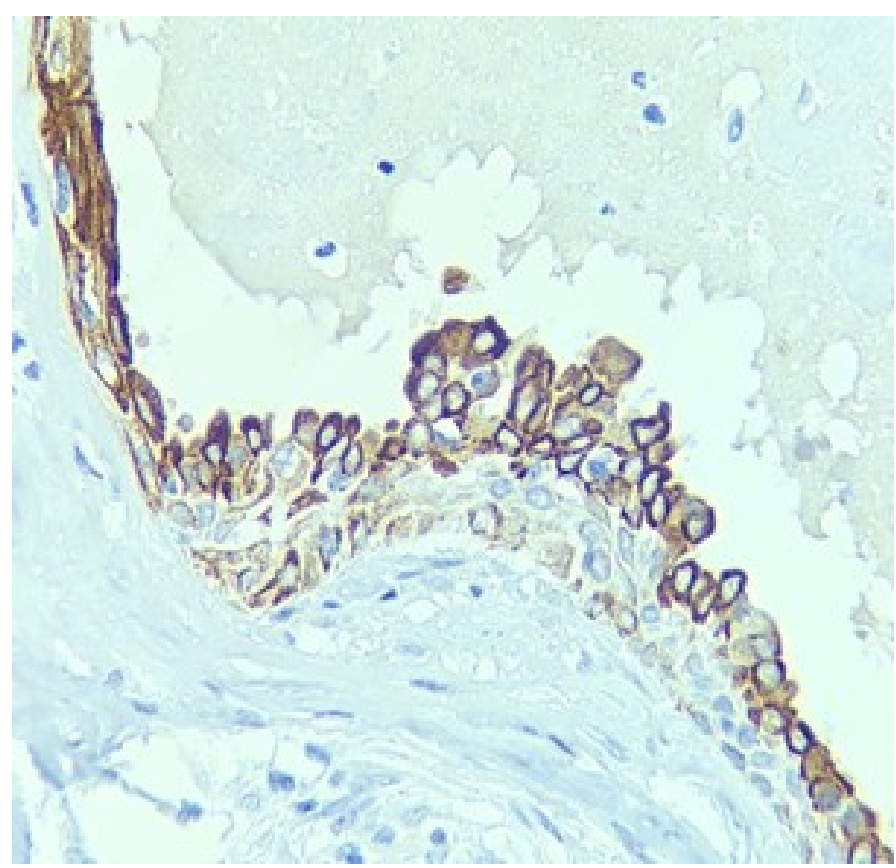

Figure 4. Cytokeratin 7 positive

When a patient develops this kind of tumor, we should claim that it is not a metastasis from distant organs (pancreas, ovary appendiceal cancer). The usual immunohistochemical profiles, to rule out metastasis from distant mucinous cystadenocarcinoma are the following: CK7 positive, CK20 negative, and CDX-2 negative, as the present case. These results can help to exclude the possibility of metastatic mucinous cystadenocarcinoma from the ovary, pancreas, and gastrointestinal tract. If we have extra resources desirable requests, MUC5 and MUC2. Those are very useful in the diagnosis of mucinous cystadenocarcinoma of the breast; MUC5 positive and MUC2 negative support this diagnosis [4]. 


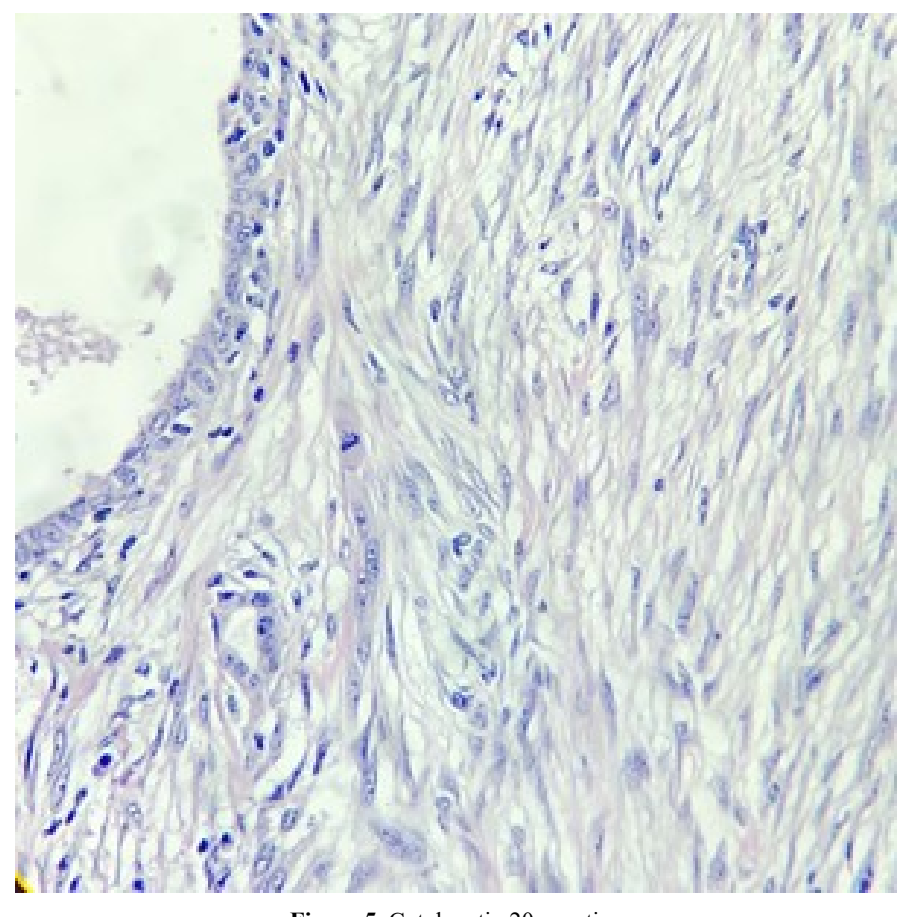

Figure 5. Cytokeratin 20 negative

Mucinous cystadenocarcinoma in the breast, together with the immune profiles of being CK7 positive, CK20 negative, CDX2 negative, estrogen receptor-negative, and progestogen receptornegative, reinforce the diagnosis [5].

This mucinous profile is different from ordinary mucinous carcinoma arising in the breast and other organs.

In medical literature, almost always this tumor is reported like case reports or small series, in the same way as the present report $[6,7]$.

Overall these tumors have a variable size since $0.8 \mathrm{~cm}$ until $19 \mathrm{~cm}$, consistency firmness to hard, in postmenopausal women, between 47 and 96 years [7].

In this case mucinous cystadenocarcinoma in the breast associated with a low-grade phyllodes tumor is reported. Two cases with an epithelial tumor have been reported, but with phyllodes, the tumor is the first one to be disclosed $[8,9]$.

The biological behavior of mucinous cystadenocarcinoma of the breast is reportedly favorable despite having a high proliferation index and triple-negative biomarker status.

The incidence of lymph node metastasis is low with only 4 cases reported in the past having macrometastasis in ipsilateral axillary lymph nodes [10].

Associated ductal carcinoma in situ with or without mucin production in 14 cases and loss of expression of estrogen receptor and progestogen receptor supports the theory of metaplastic differentiation in these cancers [11].

Other cases have been reported with nodal metastasis, with treatment with radiotherapy and chemotherapy [12]. Even with mortality by a progression of breast disease.

Most of the descriptions accomplish treatment since lumpectomy until mastectomy, with or without sentinel node biopsy, this approach is possible when we have a precise histopathological report of mucinous cystadenocarcinoma. Because of the rarity of the tumor, the histogenesis and biological behavior of this neoplasm is uncertain, but unaggressive is constantly reported [12].

The biological behavior of mucinous cystadenocarcinoma of the breast is reportedly favorable despite having a high proliferation index and triple-negative biomarker status [13].

Another noteworthy point is that although the present case originally is a combination between phyllodes and, mucinous cystadenocarcinoma. We have to wait and look if the patient develops recurrence or not, and what type of histological part, this could be epithelial or phyllodes. Nayak reported relapse of mucinous cystadenocarcinoma with ductal and component. But it was a purely mucinous element of the progressive tumor [14]

\section{Conclusion}

A proper histological diagnosis and a special mention of the rare histologic subtypes are required to formulate clear recommendations of their treatment protocols. In the next decade with a routinary molecular evaluation could be possible to propose tailor treatments not only with drugs maybe surgical treatments as well.

\section{References}

1. Koenig C, Tavassoli FA (1998) Mucinous cystadenocarcinoma of the breast Am J Surg Pathol 22: 698-703.

2. Kim SE, Park JH, Hong S, Koo JS, Jeong J, et al. (2012) Primary mucinous cystadenocarcinoma of the breast: Cytologic finding and expression of MUC5 are different from mucinous carcinoma. Korean J Pathol 46: 611-616.

3. Rosen PP (2009) Rosen's breast pathology. 3rd ed. Philadelphia: Wolters Kluwer Lippincott Williams \& Wilkins pp. 515-534

4. Matsukita S, Nomoto M, Kitajima S, Tanaka S (2003) Expression of mucins (MUC1, MUC2, MUC5AC and MUC6) in mucinous carcinoma of the breast: comparison with invasive ductal carcinoma. Histopathology 42: 26-36.

5. Hanski C, Hofmeier M, Schmitt-Gräff A, Riede E, Hanski ML, et al. (1997) Overexpression or ectopic expression of MUC2 is the common property of mucinous carcinomas of the colon, pancreas, breast, and ovary. J Pathol 182: 385-391.

6. Sentani K, Tashiro T, Uraoka N, Aosaki Y, Yano S, et al. (2012) Primary mammary mucinous cystadenocarcinoma: cytological and histological findings. Diagn Cytopathol 40: 624-628.

7. Rakici S, Gonullu G, Gursel SB, Yildiz L, Bayrak IK, et al. (2009) Mucinous cystadenocarcinoma of the breast with estrogen receptor expression: A case report and review of the literature. Case Rep Oncol 2: 210-216.

8. Chen WY, Chen CS, Chen HC, Hung YJ, Chu JS (2004) Mucinous cystadenocarcinoma of the breast co-existing with infiltrating ductal carcinoma. Pathol Int 54: 781-786.

9. Lee SH, Chaung CR (2008) Mucinous metaplasia of breast carcinoma with macrocystic transformation resembling ovarian mucinous cystadenocarcinoma in a case of synchronous bilateral infiltrating ductal carcinoma. Pathol Int 58: 601-605.

10. Koufopoulos N, Goudeli C, Syrios J, Filopoulos E, Khaldi L (2017) Mucinous cystadenocarcinoma of the breast: The challenge of diagnosing a rare entity. Rare Tumors 9:7016.

11. Honma N, Sakamoto G, Ikenaga M, Kuroiwa K, Younes M, et al. (2003) Mucinous cystadenocarcinoma of the breast: A case report and review of the literature. Arch Pathol Lab Med 127:1031-1033.

12. Nisa Z, Barman N, Dasgupta S, Pal M, Sarkar R (2015) Mucinous cystadenocarcinoma of the breast. JDP 10: 34-37.

13. Kaur M, Kaur K, Singla N (2019) Rare breast malignancy subtypes: A cytological, histological, and immunohistochemical correlation. Niger J Surg 25: 70-75.

14. Nayak A, Bleiweiss J, Dumoff K, Bhuiya TA (2018) Mucinous cystadenocarcinoma of the breast: Report of 2 cases including one with long-term local recurrence. Int J Surg Pathol 26: 1-9.

Copyright: (C2019 Valdespino VE. This is an open-access article distributed under the terms of the Creative Commons Attribution License, which permits unrestricted use, distribution, and reproduction in any medium, provided the original author and source are credited. 